\title{
Sterile Neutrino Overview
}

\author{
Jiajie Ling* \\ Sun Yat-Sen University, 135 Xingang Xi Road, Guangzhou, Guangdong, 510275, China \\ E-mail: lingjj5@mail. sysu.edu.cn
}

The basic theory and experimental hints for light sterile neutrinos at $\mathrm{eV}^{2}$ mass scale are reviewed. Besides the LSND/MiniBooNE, reactor and Gallium anomalies, other recent experimental results searching for light sterile neutrinos are also presented. The existence of the light sterile neutrino is still unclear due to the tension between those experiments. In the next a few years, several worldwide short-baseline neutrino oscillation experiments will start taking data. Hopefully the mystery of sterile neutrino will be unveiled in the near future.

The 15th International Conference on Flavor Physics \& CP Violation

5-9 June 2017

Prague, Czech Republic

${ }^{*}$ Speaker. 


\section{Introduction}

The convincing results from solar, atmospheric, reactor and long-baseline neutrino oscillation experiments in the last two decades undoubtedly demonstrate that Neutrinos having masses and large mixing [1], which is clearly beyond the standard model prediction. The simplest way to extend the Standard Model to include the neutrino masses is to introduce "right-handed neutrino" fields, which are $\mathrm{SU}(2)_{L}$ singlets and will not participate the standard weak interactions except those mixing with the active neutrinos. Based on the LEP measurements [2], there are only three types of active light neutrinos as $v_{e}, v_{\mu}$ and $v_{\tau}$. Any additional flavors of neutrinos are traditionally called sterile neutrinos [3].

Sterile neutrinos are naturally present in many theories beyond the standard model. Very heavy sterile neutrinos are introduced in the see-saw mechanism to explain the lightness of the active neutrinos. $\mathrm{KeV}$ mass scale sterile neutrinos are the candidates for warm dark matter. Sterile neutrinos at $\mathrm{eV}$ or sub-eV mass scale can alter the neutrino oscillatory behavior based on the standard $3 \times 3$ PMNS matrix [4, 5]. They are the candidates account for the LSND/MiniBooNE [6, 8], reactor [9] and Gallium [14] anomalies. In this review, we will focus on the $\mathrm{eV}^{2}$ mass scale light sterile neutrinos.

In the simplest $3+1$ active-sterile neutrino mixing framework, three active neutrinos flavor $v_{e}$, $v_{\mu}, v_{\tau}$ are mainly mixed with three light neutrino mass eighenstates $v_{1}, v_{2}, v_{3}$, and one sterile neutrino $v_{s}$ is mainly mixed with $\mathrm{eV}$ scale neutrino mass eigenstate $v_{4}$, as shown in Fig 1 .

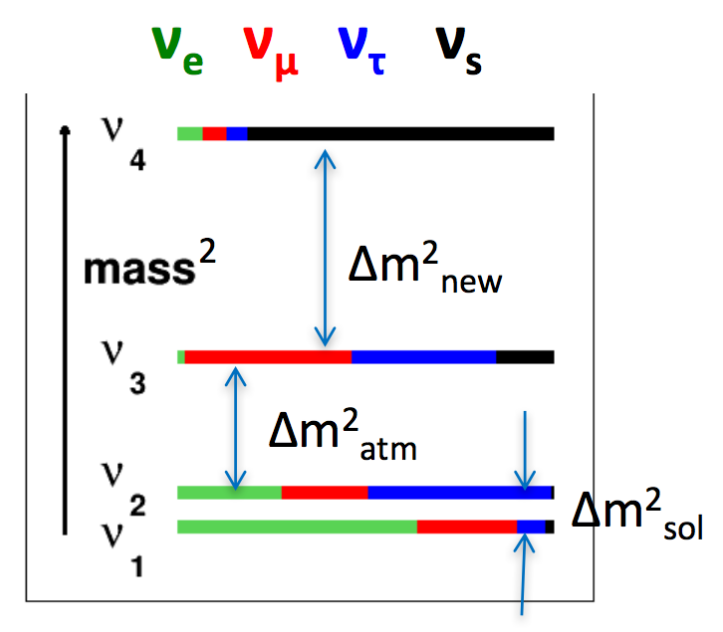

Figure 1: The $3+1$ active-sterile neutrino mixing.

Since the large squared-mass difference between $m_{1,2,3}^{2}$ and $m_{4}^{2}, \Delta m_{\text {new }}^{2} \gg \Delta m_{\text {atm }}^{2} \gg \Delta m_{\text {sol }}^{2}$, three light neutrino mass eigenstates consolidate as one mass eigenstate. The short baseline neutrino oscillation can be treated as 2-neutrino oscillation modes:

$$
\begin{aligned}
P\left(v_{\alpha} \rightarrow v_{\beta}\right) & \cong 4\left|U_{\alpha 4}\right|^{2}\left|U_{\beta 4}\right|^{2} \sin ^{2}\left(\frac{\Delta m_{41}^{2} L}{4 E}\right) \\
& \cong \sin ^{2} 2 \theta_{\alpha \beta} \sin ^{2}\left(\frac{\Delta m_{41}^{2} L}{4 E}\right)
\end{aligned}
$$




$$
\begin{aligned}
P\left(v_{\alpha} \rightarrow v_{\alpha}\right) & \cong 1-4\left|U_{\alpha 4}\right|^{2}\left(1-\left|U_{\alpha 4}\right|^{2}\right) \sin ^{2}\left(\frac{\Delta m_{41}^{2} L}{4 E}\right) \\
& \cong 1-\sin ^{2} 2 \theta_{\alpha \alpha} \sin ^{2}\left(\frac{\Delta m_{41}^{2} L}{4 E}\right)
\end{aligned}
$$

For $\left|U_{\alpha 4}\right|^{2}$ and $\left|U_{\beta 4}\right|^{2}$ being small, $\sin ^{2} 2 \theta_{\alpha \alpha} \approx 4\left|U_{\alpha 4}\right|^{2}, \sin ^{2} 2 \theta_{\beta \beta} \approx 4\left|U_{\beta 4}\right|^{2}$,

$$
\sin ^{2} 2 \theta_{\alpha \beta} \approx 4\left|U_{\alpha 4}\right|^{2}\left|U_{\beta 4}\right|^{2} \approx \frac{1}{4} \sin ^{2} 2 \theta_{\alpha \alpha} \sin ^{2} 2 \theta_{\beta \beta}
$$

Besides $3+1$ active-sterile neutrino mixing model, two or more massive sterile neutrinos can be added into the neutrino mixing matrix, known as $3+2,1+3+1$ or other more complicated models. Due to the degeneracy of the three light neutrino mass eigenstates, the short baseline neutrino oscillation can be simplified as 3-neutrino oscillation for $3+2$ model, which introduces a CP-violation phase to break down the symmetry between neutrino and anti-neutrino oscillation probabilities.

\section{Experimental results on sterile neutrinos}

The LSND anomaly, the reactor antineutrino anomaly and the Gallium neutrino anomaly are the three experimental hints of short-baseline neutrino oscillations, which require at least one additional heavy neutrino with mass square difference $\Delta m_{\text {new }}^{2} \sim 1 \mathrm{eV}^{2}$, much larger than $\Delta m_{\text {atm }}^{2}$ and $\Delta m_{\text {sol }}^{2}$. In the meanwhile, those results are in tension with the other experimental results with null sterile neutrino signals.

\subsection{The LSND anomaly and other short-baseline neutrino appearance experiments}

The Liquid Scintillator Neutrino Detector (LSND) experiment [6] is a short-baseline acceleratorbased neutrino oscillation experiment, aiming to search $\bar{v}_{\mu} \rightarrow \bar{v}_{e}$ oscillation. LSND utilized 798 $\mathrm{MeV}$ proton from the LAMPF accelerator impinging the water and later on replaced with a closepacked, high-Z target, to produce the stopping $\pi^{+}$and $\mu^{+}$, which generate the $v_{\mu}, \bar{v}_{\mu}$ and $v_{e}$ flux with decay-at-rest mode. The $v_{e}$ events are detected by a 167 -ton liquid scintillator detector located at 30 meters from the neutrino source through inverse-beta-decay process. The selected positron energy is in the range of 20 to $60 \mathrm{MeV}$.

With a clean selected oscillation sample, the beam on-off excess is $49.1 \pm 9.4$ events. Two major beam-related neutrino backgrounds are $16.9 \pm 2.3$ events, which mainly contribute from $\bar{v}_{e}$ from $\mu^{-}$decay-at-rest in the beam stop, and $\bar{v}_{\mu}$ from $\pi^{-}$decay-in-flight. This $32.2 \pm 9.4 \pm 2.3 \bar{v}_{e}$ appearance signal corresponds to $3.8 \sigma$, which is well fitted with $v_{\mu} \rightarrow v_{e}$ oscillation at $>0.2 \mathrm{eV}^{2}$ squared-mass difference, given the $\mathrm{L} / \mathrm{E} \sim 1 \mathrm{~m} / \mathrm{MeV}$.

However, the KARMEN experiment [7], which also uses the stopping pion and muon technique based on $800 \mathrm{MeV}$ protons pulsed beam from the ISIS facility in RAL, does not observe such evidence of $\bar{v}_{\mu} \rightarrow \bar{v}_{e}$ oscillation at $17.7 \mathrm{~m}$ baseline. KARMEN observes 15 candidate events, which is consistent with the estimated background of $15.8 \pm 0.5$ events. Since the baseline of KARMEN is shorter than LSND, the signal region of the LSND can not be totally excluded by KARMEN.

In order to cross check the LSND result, the MiniBooNE experiment [8] is carried out in FNAL with the similar $\mathrm{L} / \mathrm{E}$. The pulsed $8 \mathrm{GeV}$ proton beams from Booster, hitting on the beryllium target, 
generate pion and kaons, which will decay to the $v_{\mu}$ and $\bar{v}_{\mu}$ neutrino flux in flight. The baseline of the MiniBooNE experiment is $500 \mathrm{~m}$ and the peak energy is about $500 \mathrm{MeV}$. By switching the polarity of magnetic horns, MiniBooNE runs the experiment on neutrino or antineutrino oscillation search modes seperately. For $v_{\mu} \rightarrow v_{e}$ mode: MiniBooNE observes $162.0 \pm 47.8$ events excess. However, most of the electron-like events excess is for $E_{v}<475 \mathrm{MeV}$, which are at a different $L / E$ region from LSND signals. For $\bar{v}_{\mu} \rightarrow \bar{v}_{e}$ mode: $78.4 \pm 28.5$ event excess is observed. The event excess for $E_{v} L 475 \mathrm{MeV}$ is consistent with the LSND signal. As shown in Fig 2, a more complicated $3+2$ active-sterile neutrino mixing framework is introduced to explain the difference between neutrino and antineutrino oscillation modes through the $\mathrm{CP}$-violating term.
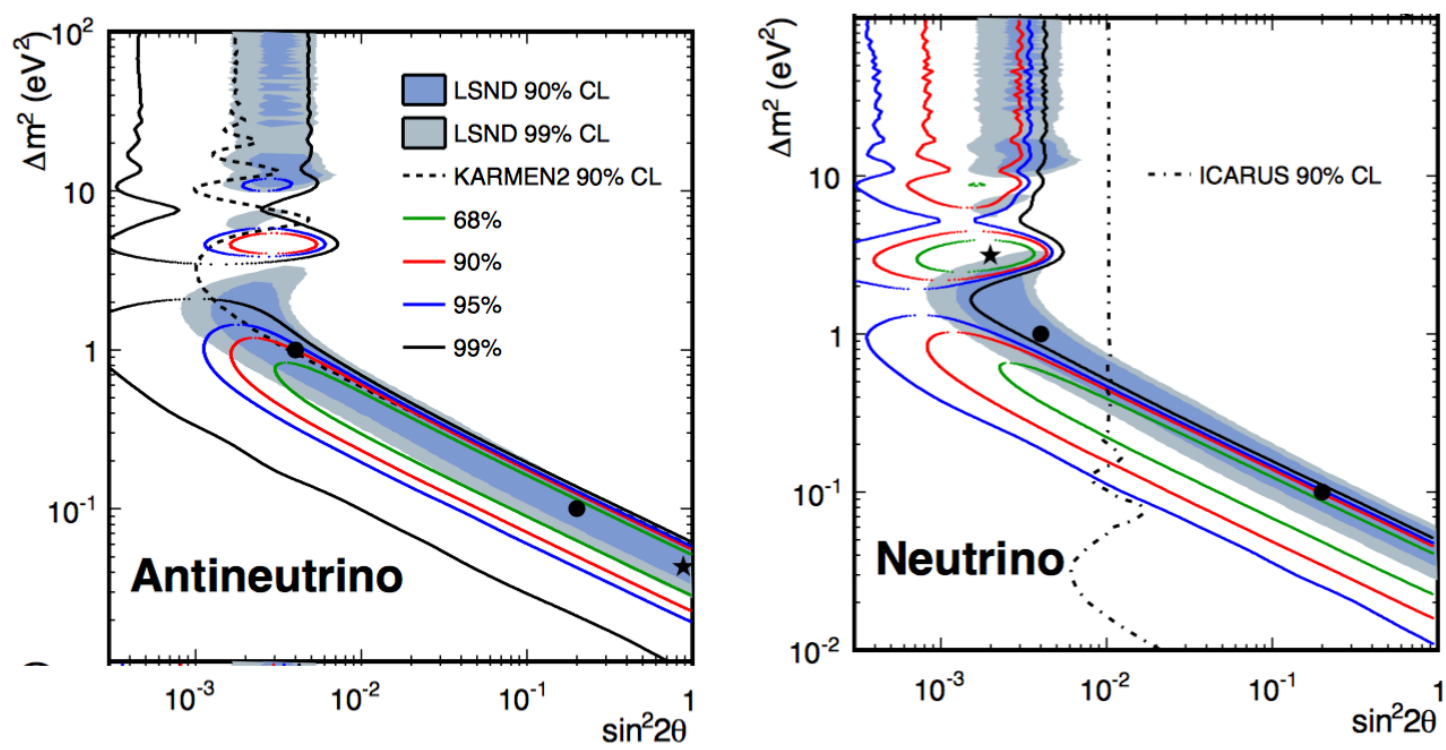

Figure 2: The MiniBooNE results on $\bar{v}_{\mu} \rightarrow \bar{v}_{e}$ and $v_{\mu} \rightarrow v_{e}$ oscillation results

\subsection{The reactor antineutrino anomaly and Gallium neutrino anomaly}

Another hint of the existence of sterile neutrinos comes from the updated reevaluation of reactor antineutrino fluxes, which predict $\sim 5 \%$ more antineutrino flux than the previous one. In addition, the IBD cross section is increased by $1 \%$ due to the recent smaller measured neutron lifetime. As shown in Fig. 3, the overall predicted reactor antineutrino events is about $6 \%$ more than the experimental data measured at baseline from 20 to 100 meters. The uncertainty of the predicted reactor flux is about $2 \%[10,11]$, the statistical significance of the reactor antineutrino anomaly is about $3 \sigma$ [9]. This event deficit could also be explained with sterile neutrino $\bar{v}_{e} \rightarrow \bar{v}_{e}$ oscillation with squared-mass difference $\Delta m^{2} \sim 1 \mathrm{eV}^{2}$. Another possible explanation of the reactor antineutrino anomaly could be the underestimation of the reactor antineutrino flux prediction [32]. Some study shows the reactor antineutrino flux uncertainty could be larger than $4 \%$ due to the unknown forbidden decays in hundreds of nuclei beta decay processes [15]. Especially the observation of an excess of antineutrino spectrum at 5-7 MeV region in Daya Bay [25], RENO [27] and Double Chooz [26] experiments, gives some evidence for the inaccuracy of the reactor antineutrino flux prediction. The Daya Bay measurement is shown in Fig. 4(a). 


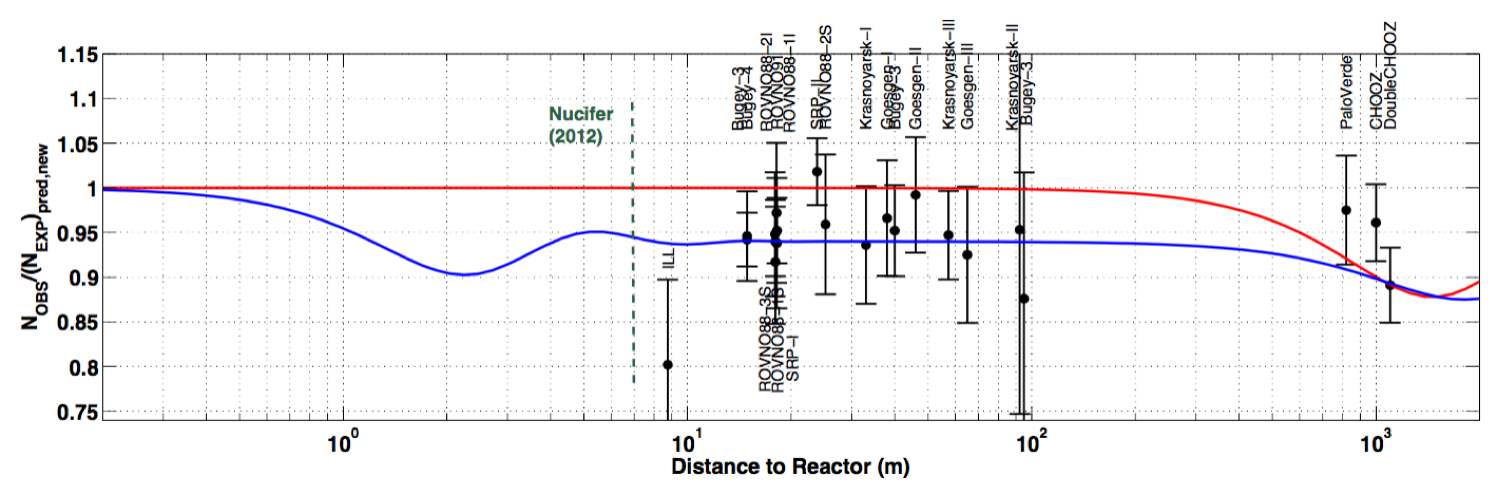

Figure 3: The reactor antineutrino anomaly

Recently Daya Bay experiment presented the results of the measurement of the correlation between the reactor fuel evolution and the changes in the antineutrino flux [28]. Using detector data spanning effective ${ }^{239} \mathrm{Pu}$ fission fraction $F_{239}$ from 0.25 to 0.35 , the measured evolution in total IBD yield disagree with the predictions from the reactor model at 2.7 $\sigma$. As shown in Fig. 4(b), this discrepancy indicates the overall deficit in the measured flux with repect to predictions mainly comes from ${ }^{235} U$ instead of the active-sterile neutrino oscillation hypothesis, which will cause equal fractional deficits from the primary fission isotopes ${ }^{235} U,{ }^{239} \mathrm{Pu},{ }^{238} \mathrm{U}$ and ${ }^{241} \mathrm{Pu}$. In the meanwhile, the Daya Bay evolution data and global reactor data can be well-fitted with composite hypotheses including variations of ${ }^{235} \mathrm{U}$ or ${ }^{239} \mathrm{Pu}$ fluxes in addition to active-sterile neutrino oscillations [30, 29]. And it is also pointed out that the antineutrino spectra through the ab initio method which sums over all fission fragment beta-decays using nuclear database input, can explains all the features in the Daya Bay evolution data. However, the reactor antineutrino anomaly still exist [31].

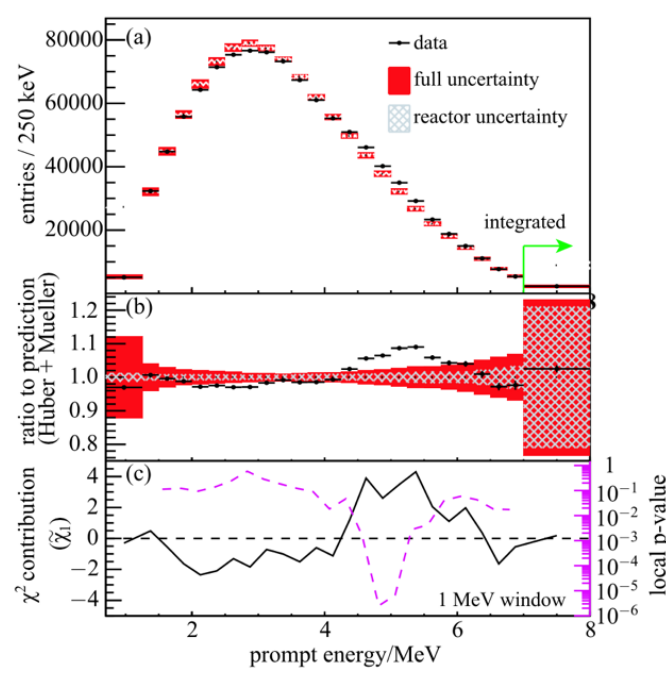

(a)

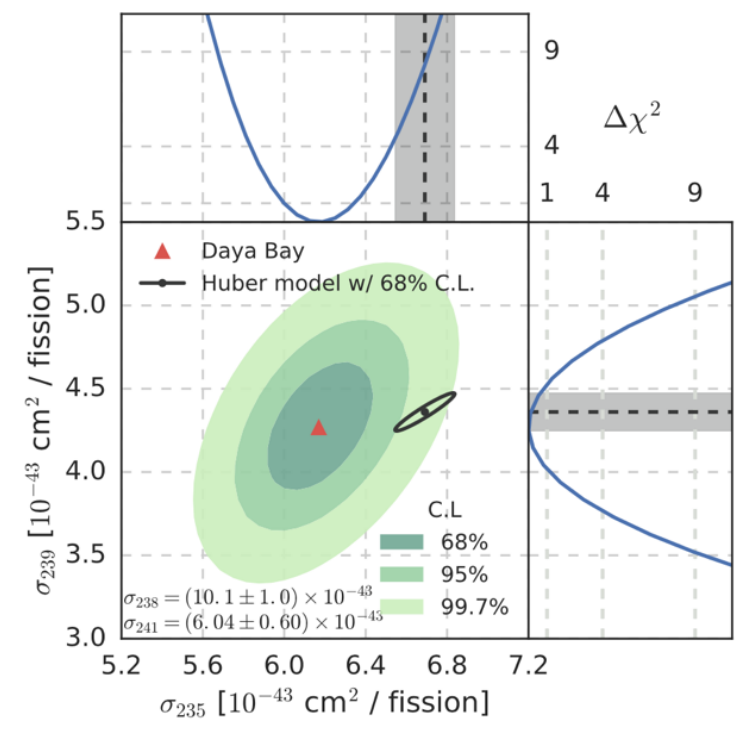

(b)

Figure 4: (a) The "bump" structure observed in reactor antineutrino spectrum. (b) Daya Bay's reactor fuel evolution results on the IBD yields from ${ }^{239} \mathrm{Pu}$ and ${ }^{235} U$. 
The third hints of sterile neutrino is called Gallium neutrino anomaly [14], which observed short-baseline disappearance of $v_{e}$ in two solar neutrino experiments, GALLEX [12] and SAGGE [13], using Gallium radioactive sources deployed inside the detectors. The averaged ratio of the measured events to the predicted events $\bar{R}=0.84 \pm 0.05$ with statistical significance of $\sim 2.9 \sigma$. This is consistent with the reactor antineutrino anomaly assuming CPT symmetry conservation.

\subsection{Sterile neutrino searching from other experiments}

Besides the global analysis hunting for $\mathrm{eV}^{2}$ mass-squared difference active-sterile neutrino mixing, where the fast oscillation causes the integrated reactor antineutrino flux deficit for experiments at baselines of 20-100 meters, sub-eV $\mathrm{V}^{2}$ mass-squared active-sterile neutrino mixing can also distort the reactor antineutrino spectrum.

Bugey-3 experiment [18] uses a segmented ${ }^{6} \mathrm{Li}$-doped liquid scintillator detector located at 15 , 40 and $95 \mathrm{~m}$ baselines from the reactors to measure the sterile neutrino oscillation. No spectrum distortion is observed for those three distances.

Recently NEOS experiment [19] also measures the reactor antineutrino flux from the $2.8 \mathrm{GW}_{t h}$ Hanbit nuclear power plant in Yeonggwang, Korea. The reactor core is cylindrical shape with size of $3.1 \mathrm{~m}$ diameter and $3.8 \mathrm{~m}$ height. NEOS uses a monolithic Gd-LS detector at baseline of 23.7 $\mathrm{m}$ from the reactor. No spectrum distortion is observed and the result is consistent with Bugey-3 experiment.

The Daya Bay experiment [20] has eight Gd-LS antineutrino detectors located in three experimental halls close to the Daya Bay reactor power plant complex with total thermal power of 17.4 GW. The baselines are from $365 \mathrm{~m}$ to about $2 \mathrm{~km}$. By comparing the reactor antineutrino spectra of detectors at various baselines, Daya Bay excludes the active-sterile neutrino mixing at $10^{-3} \mathrm{eV}^{2}<\Delta m^{2}<0.2 \mathrm{eV}^{2}$ region.
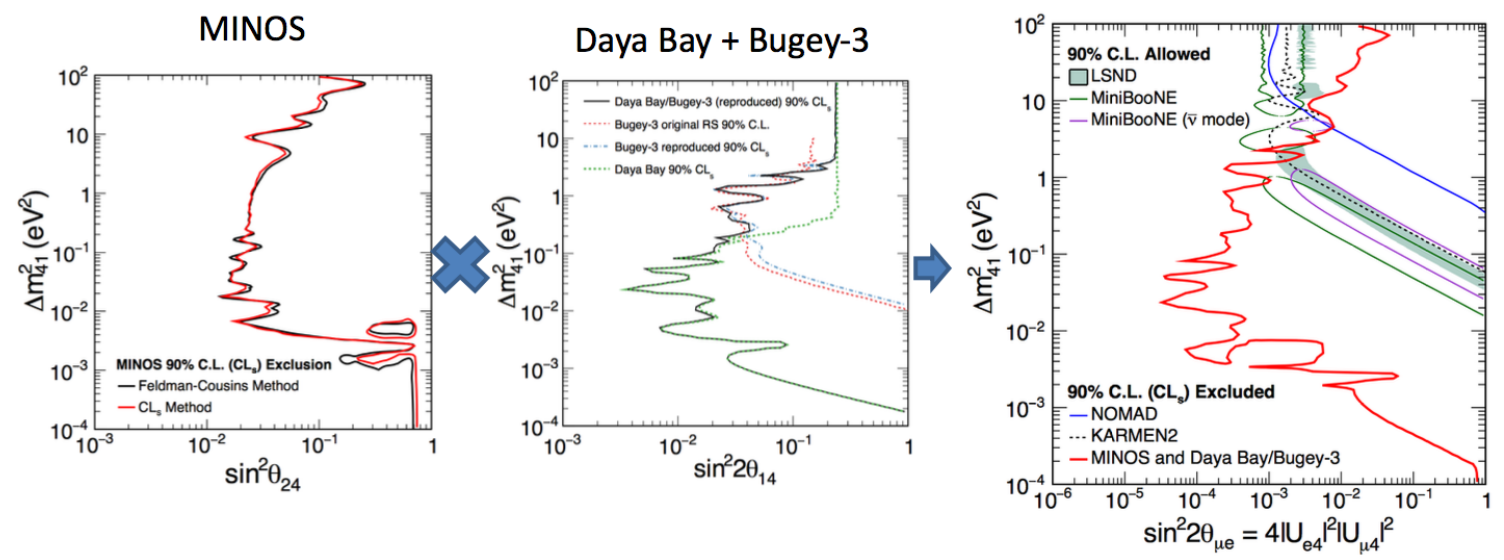

Figure 5: The sterile neutrino search results of $v_{\mu} \rightarrow v_{\mu}$ from MINOS, and $\bar{v}_{e} \rightarrow \bar{v}_{e}$ from Daya Bay and Bugey-3, and the joint analysis on constraining $\sin ^{2} 2 \theta_{\mu e}$

In the meanwhile, the sterile neutrino searches are also conducted in the muon neutrino disappearance channel, including Super-K, CDHS, CCFR, SciBooNE and MiniBooNE experiment [23]. None of them observe the sterile neutrino oscillation. Recently MINOS [16] and IceCube [17] both reported their result on sterile neutrino searches. No sterile neutrino hints are found. As shown in 
Fig. 5, a joint analysis of Daya Bay, Bugey-3 and MINOS sterile neutrino analysis is able to largely exclude the LSND and MiniBooNE anomaly signal region based on 3+1 active-sterile neutrino mixing model [21].

Clearly there is a tension between neutrino appearance and disappearance results on the sterile neutrino searches. It is also shown in the global analysis which combines the $v_{\mu} \rightarrow v_{m u}$ and $v_{e} \rightarrow v_{e}$ short-baseline data and compare with $v_{\mu} \rightarrow v_{e}$ results [23, 22].

The current cosmology data [24] can also give the constraints on the types and neutrino mass. The number of relativistic species of neutrino $N_{\text {eff }}=3.15 \pm 0.23$, which is consistent with the standard model prediction $N_{e f f}=3.046$. The total sum of the neutrino mass $\sum_{i} m\left(v_{i}\right)<0.23 \mathrm{eV}$.

\section{Future short-baseline neutrino Experiments}

There are many interesting ideas and experiments are designed or ongoing for hunting the sterile neutrinos. In order to give a convincing evidence of the existence of the sterile neutrino oscillation, the next generation sterile neutrino experiments need to observe the fast oscillation distortion on the neutrino spectrum besides the integral neutrino flux deficit from neutrino disappearance experiments. They can be roughly categorized into several approaches, including strong radioactive neutrino/antineutrino sources experiments, short baseline reactor neutrino experiments, stopping $\pi / \mu$ beam experiments and decay-in-flight beam experiment.

\subsection{Radioactive neutrino/antineutrino source experiments}

Deploying a strong radioactive neutrino or antineutrino source inside or outside of a large scintillator detector, which has superb energy resolution and vertex reconstruction, can effectively observe the sterile neutrino oscillation pattern inside the detector. A great benefit of such experiment is that the size of the radioactive source is negligible. However, the difficulty of such experiment is the source production and shipment. One of the most promising such experiments is the SOX experiment [33], which is plan to deploy a $100-150 \mathrm{kCi}{ }^{144} \mathrm{Ce} \bar{v}_{e}$ source beneath the Borexino detector at a baseline of $8.5 \mathrm{~m}$ from the detector center. They expect to start the data-taking around 2018. With roughly 1.5 years of data taking, the sensitivity of the SOX rate and spectrum analysis is able to exclude most of the reactor anomaly signal region, which is shown in Fig. 6(a).

\subsection{Short baseline reactor experiments}

Various short baseline reactor antineutrino experiments around the world try to search for sterile neutrinos at baseline of 10s meters, including DANSS and Neutrino4 in Russia, NEOS in South Korea, PROSPECT, nuLat, Chandler in U.S.A, Solid in Belgium and Stereo in France. Most of them use segmented Gd or Lithium doped scintillator detector with pulse shape discrimination to enable better vertex reconstruction, thermal neutron capture enlargement and fast neutron reduction.

\subsection{Stopping $\pi / \mu$ beam experiments}

JSNS/J-PARC E56 [34] is a stopping $\pi / \mu$ beam experiment located in J-PARC about $24 \mathrm{~m}$ from spallation neutron target in order to direct test on the LSND anomaly. Since it uses the $3 \mathrm{GeV}$ 
pulsed JSNS proton beam, it can largely reduce the beam-off background. The experiment will start with a $50 \mathrm{t} \mathrm{Gd-doped} \mathrm{liquid} \mathrm{scintillator} \mathrm{detector} \mathrm{construction} \mathrm{with} \mathrm{the} \mathrm{grant-in-aid} \mathrm{approval} \mathrm{in}$ JFY2018.

\subsection{Decay-in-flight beam experiments}

The short-baseline neutrino program [35] at FNAL will use the liquid Argon Time Projection Chamber technology to detect the neutrino generated by FNAL Booster $8 \mathrm{Gev}$ protons. It has SBND (112 ton), MicroBooNE (87 ton) and ICARUS (476 ton) three detectors at baselines of $110 \mathrm{~m}, 470 \mathrm{~m}$ and $600 \mathrm{~m}$ from the target. Currently MicroBooNE already recorded $3.4 \times 10^{20}$ POT. ICARUS and SBND is under civil reconstruction and will be ready in 2018-2019. As shown in Fig. $6(\mathrm{~b})$, with $6.6 \times 10^{20}$ POT, the sensitivity is able to exclude the LSND/MiniBooNE signal region with $5 \sigma$.

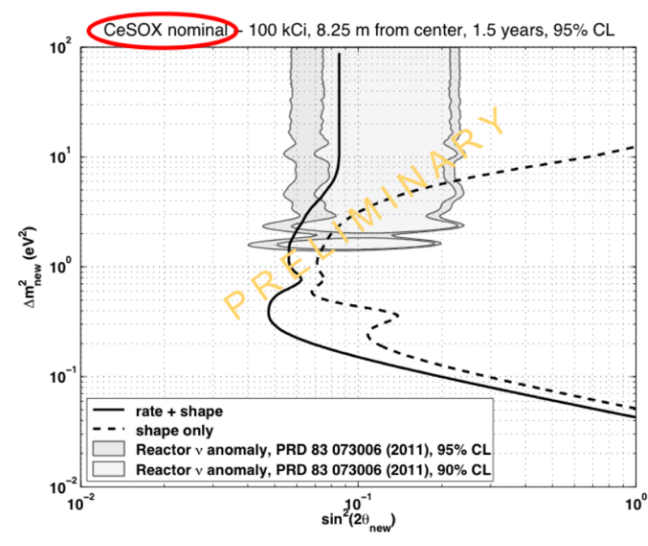

(a)

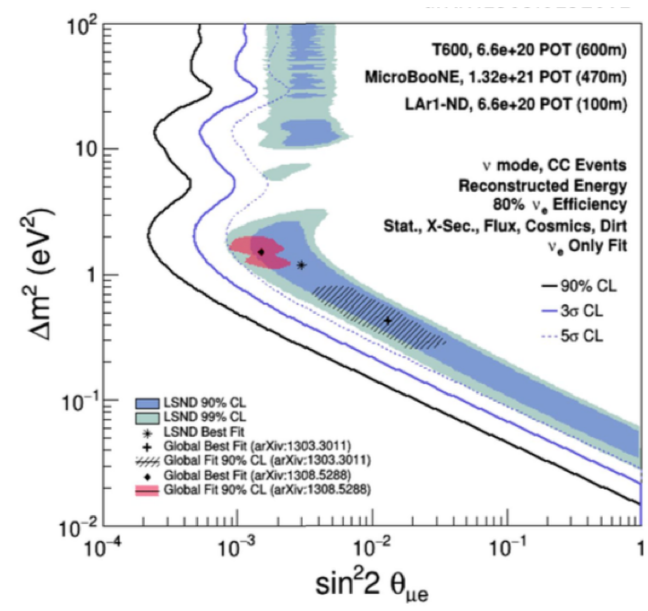

(b)

Figure 6: (a) The sensitivity of SOX experiment on $\bar{v}_{e}$ disappearance (b) The sensitivity of SBN experiment on $v_{\mu} \rightarrow v_{e}$ appearance

\section{Summary}

The existence of sterile neutrinos is still a mystery and worth to explore. There are some experimental hints of the existence of the sterile neutrinos, however, not conclusive. Given many sterile neutrino searches are on-going or planned, hopefully a definitive answer on the existence of $\mathrm{eV}$ scale sterile neutrinos are expected in the next few years.

\section{References}

[1] C. Patrignani et al. (Particle Data Group), Chin. Phys. C 40, 100001 (2016).

[2] S. Schael, et al. (ALEPH Collaboration, DELPHI Collaboration, L3 Collaboration, OPAL Collaboration, SLD Collaboration, LEP Electroweak Working Group, SLD Electroweak Group, SLD Heavy Flavour Group), Phys. Rept.427, 257 (2006) [hep-ex/ 0509008 ]. 
[3] K. N. Abazajian, et al., [hep-ph/1204.5379] (2012)

[4] B. Pontecorvo, Sov. Phys. JETP 26, 984 (1968).

[5] Z. Maki, M. Nakagawa, and S. Sakata, Prog. Theor. Phys. 28, 870 (1962).

[6] A. Aguilar, et al. (LSND Collaboration), Phys. Rev. D 64, 112007 (2001) [hep-ex/ 0104049 ].

[7] B. Armbruster, et al. (KARMEN Collaboration), Phys. Rev. D 65, 112001 (2002) [hep-ex/0203021].

[8] A. Aguilar-Arevalo, et al. (MiniBooNE Collaboration), Phys. Rev. Lett. 110, 161801 (2013) [hep-ex/1303.2588].

[9] G. Mention, M. Fechner, T. Lasserre, T. A. Mueller, D. Lhuil- lier, M. Cribier, and A. Letourneau, Phys. Rev. D 83, 073006 (2011) [hep-ex/1101.2755].

[10] T. A. Mueller, D. Lhuillier, M. Fallot, A. Letourneau, S. Cor- mon, L. Fechner, M. Giot, T. Lasserre, J. Martino, G. Mention, et al., Phys. Rev. C 83, 054615 (2011) [hep-ex/1101.2663]

[11] P. Huber, Phys. Rev. C 84, 024617 (2011) [hep-ph/1106.0687].

[12] W. Hampel et al. (GALLEX Collaboration), Phys. Lett. B420, 114 (1998).

[13] J. Abdurashitov et al. (SAGE Collaboration), Phys. Rev. C 80, 015807 (2009) [nucl-ex/0901.2200].

[14] C. Giunti and M. Laveder, Phys. Rev. C 83, 065504 (2011) [hep-ph/1006. 3244 ].

[15] A. Hayes, J. Friar, G. Garvey, G. Jungman, and G. Jonkmans, Phys. Rev. Lett. 112, 202501 (2014) [nucl-th/1309.4146].

[16] P. Adamson, et al. (MINOS Collaboration), Phys. Rev. Lett. 117, 151803 (2016) [hep-ex/1607.01176].

[17] M. G. Aartsen et al. [IceCube Collaboration], Phys. Rev. D 95, no. 11, 112002 (2017) [hep-ex/1702.05160].

[18] Y. Declais, et al. (Bugey Collaboration), Nucl. Phys. B 434, 503 (1995).

[19] Y. J. Ko et al., Phys. Rev. Lett. 118, no. 12, 121802 (2017) [hep-ex/1610 . 05134].

[20] F.P. An, et al. (Daya Bay Collaboration), phys. Rev. Lett. 117, 151802 (2016) [hep-ex/1607.01174].

[21] P. Adamson, et al. (Daya Bay, MINOS Collaboration), phys. Rev. Lett. 117, 151801 (2016) [hep-ex/1607.01177].

[22] S. Gariazzo, C. Giunti, M. Laveder and Y. F. Li, JHEP 1706, 135 (2017) [hep-ph/1703. 00860 ].

[23] J. Kopp, P. A. N. Machado, M. Maltoni and T. Schwetz, JHEP 1305, 050 (2013) [hep-ph/1303.3011].

[24] P. A. R. Ade et al. [Planck Collaboration], Astron. Astrophys. 594, A13 (2016) [astro-ph.co/1502.01589].

[25] F.P. An, et al. (Daya Bay Collaboration), Chin. Phys. C 41, 013002 (2017) [hep-ex/1607.05378].

[26] Y. Abe, et al. (Double Chooz Collaboration), J. High Energy Phys. 10 (2014) 086.

[27] S.-H. Seo (RENO Collaboration), AIP Conf. Proc. 1666, 080002 (2015). 
[28] F.P. An, et al. (Daya Bay Collaboration), phys. Rev. Lett. 118, 251801 (2017) [hep-ex/1704.01082].

[29] M. Dentler, ÃA. HernÃąndez-Cabezudo, J. Kopp, M. Maltoni and T. Schwetz, [hep-ph/1709.04294].

[30] C. Giunti, X. P. Ji, M. Laveder, Y. F. Li and B. R. Littlejohn, [hep-ph/1708.01133].

[31] A. Hayes, G. Jungman, L. McCutchan, A. Sonzogni, G. Garvey and X. Wang, [nucl-th/1707.07728].

[32] A. A. Sonzogni, E. A. McCutchan and A. C. Hayes, Phys. Rev. Lett. 119, no. 11, 112501 (2017).

[33] G. Ranucci [SOX Collaboration], PoS NOW 2016, 061 (2017).

[34] S. Ajimura et al.,[physics.ins-det/1705.08629] (2017).

[35] D. Cianci, A. Furmanski, G. Karagiorgi and M. Ross-Lonergan, Phys. Rev. D 96, no. 5, 055001 (2017) [hep-ph/1702.01758]. 